\title{
Geometric and functional architecture of visceral sensory microcircuitry
}

\author{
Yoshikatsu Negishi • Yoshinori Kawai
}

Received: 22 July 2010/Accepted: 23 November 2010/Published online: 14 December 2010

(C) The Author(s) 2010. This article is published with open access at Springerlink.com

\begin{abstract}
Is microcircuit wiring designed deterministically or probabilistically? Does geometric architecture predict functional dynamics of a given neuronal microcircuit? These questions were addressed in the visceral sensory microcircuit of the caudal nucleus of the tractus solitarius (NTS), which is generally thought to be homogeneous rather than laminar in cytoarchitecture. Using in situ hybridization histochemistry and whole-cell patch clamp recordings followed by neuronal reconstruction with biocytin filling, anatomical and functional organization of NTS microcircuitry was quantified to determine associative relationships. Morphologic and chemical features of NTS neurons displayed different patterns of process arborization and sub-nuclear localization according to neuronal types: smaller cells featured presynaptic local axons and GABAergic cells were aggregated specifically within the ventral NTS. The results suggested both a laminar organization and a spatial heterogeneity of NTS microcircuit connectivity. Geometric analysis of pre- and postsynaptic axodendritic arbor overlap of reconstructed neurons (according to parent somal distance) confirmed a heterogeneity of microcircuit connectivity that could underlie differential functional dynamics along the dorsoventral axis. Functional dynamics in terms of spontaneous and evoked postsynaptic current patterns behaved in a strongly location-specific manner according to the geometric dimension, suggesting a spatial laminar segregation of neuronal populations: a dorsal group
\end{abstract}

Electronic supplementary material The online version of this article (doi:10.1007/s00429-010-0294-5) contains supplementary material, which is available to authorized users.

Y. Negishi $(\bowtie) \cdot$ Y. Kawai

Department of Anatomy, Jikei University School of Medicine,

3-25-8 Nishi-Shimbashi Minato-ku, Tokyo 105-8461, Japan

e-mail: yoshinegishi@jikei.ac.jp of high excitation and a ventral group of balanced excitation and inhibition. Recurrent polysynaptic activity was also noted in a subpopulation of the ventral group. Such geometric and functional laminar organization seems to provide the NTS microcircuit with both reverberation capability and a differentiated projection system for appropriate computation of visceral sensory information.

Keywords Nucleus of the tractus solitarius . Laminar organization - GABAergic · Glutamatergic . Reverberation · Axon collateral

\section{Introduction}

Peripheral visceral sensory inputs are processed in the nucleus of tractus solitarius (NTS), a nucleus specialized for central transfer of visceral information, including cardiorespiratory reflexes. Functional and anatomical specificity of local synaptic connectivity within the NTS could define a substrate for information processing. Besides being a relay for information transfer, there exist significant intrinsic synaptic activities that may relate to local feedforward and feedback axon collaterals within this nucleus (Fortin and Champagnat 1993; Kawai and Senba 1996). Microcircuits formed by excitatory and inhibitory local synapses in the NTS could also underlie some aspects of reverberation and pacemaker-like activity reported to occur in this nucleus (Champagnat et al. 1985, 1986; Smith et al. 1998; Davis et al. 2004). However, the functional significance of these recurrent connections remains to be elusive. Recurrent activity generated by microcircuits has been implicated in several important functions, such as generating stochastic fluctuations for non-linear computation (Douglas and Martin 2007), eliciting synchronized 
oscillations implicated in memory consolidation ( $\mathrm{Ji}$ and Wilson 2007), and creating reverberatory activity that can serve as a substrate for working memory (Sanchez-Vives and McCormick 2000; Lau and Bi 2005). It may be too challenging to ask whether such functions could be attributed to the NTS microcircuit. However, before tackling this question, it is currently of fundamental interest to address the relationship between anatomical and functional architectures of microcircuits that could underlie intrinsic NTS synaptic activity.

Recently, neurogeometry was employed for a quantitative analysis of potential synaptic connectivity in cortical microcircuits, based on three-dimensional reconstruction of single neurons (Shepherd et al. 2005; Stepanyants and Chklovskii 2005). This method investigates axodendritic overlap of paired reconstructed neurons as a function of their spatial separations. These results combined with those from other morphometric studies of cortical synaptic connectivity have confirmed a rather probabilistic nature of connectivity, known as Peters' rule (Braitenberg and Schuz 1998). This is somewhat surprising given the seemingly deterministic design of cortical microcircuits: differentiation of pyramidal and non-pyramidal neurons, specific GABAergic synaptic targeting (e.g., exclusive axo-axonic synapses from a chandelier cell), in addition to the prominent laminar specificity of neuronal organization. Unlike the cerebral cortex, which performs complicated tasks including cognition and sensory processing, the NTS is likely to have a much simpler form of microcircuit design (Kawai and Senba 1996; Douglas and Martin 2007). With this background, there arise several questions. Is wiring of the NTS microcircuit more probabilistic or deterministic than that of the cortex? Are there any differences or similarities in microcircuit design between the cortex and the NTS? What precisely are the differences or similarities? Can intrinsic synaptic activities be associated with the microcircuit design? What specific function does the microcircuit in the NTS perform?

In our previous studies, we revealed correlations of neuronal size with other morphological and electrophysiological characteristics in the caudal NTS (cNTS) (Kawai and Senba 1996, 1999, 2000; Okada et al. 2006; Yoshioka et al. 2006). However, quantification of geometric parameters, such as relative locations of pre- and postsynaptic cells and the overlap of axonal and dendritic arborizations, were not considered in our previous correlative studies of morphology and function. In the present study, a geometric analysis based on soma location and axodendritic overlap, rather than mere quantification according to somal size, was applied to the visceral sensory microcircuitry of the NTS to address more realistically the relationship between morphological design and functional dynamics in the NTS microcircuit.

\section{Materials and methods}

Animals

Experiments were performed on Sprague-Dawley rats (Postnatal days 13-24, Saitama Experimental Animals Supply, Japan) deeply anesthetized with ether. All experiments were performed in accordance with the Guidelines on Animal Experimentation of the Jikei University School of Medicine. After decapitation at the cervical spinal level followed by a rapid craniotomy, the brainstem mass including the cerebellum was quickly removed and a 2- to 3-mm thick block containing the area postrema (caudal medulla oblongata) was prepared for coronal slicing. Usually two slices containing the area postrema for patchclamp whole-cell recordings and biocytin filling were available from each animal.

Whole-cell patch-clamp recording and stimulation

Coronal slices (250-300 $\mu \mathrm{m}$ thickness) were made by a microslicer (DTK-1000, Dosaka, Japan). Slices containing the area postrema were collected and incubated in standard Ringer's solution for at least $1 \mathrm{~h}$ at $37^{\circ} \mathrm{C}$. The standard Ringer's solution had the following composition (in $\mathrm{mM}$ ): $125 \mathrm{NaCl}, 2.5 \mathrm{KCl}, 2 \mathrm{CaCl}_{2}, 1 \mathrm{MgCl}_{2}, 1.25$ $\mathrm{NaH}_{2} \mathrm{PO}_{4}, 26 \mathrm{NaHCO}_{3}$, and 10 glucose, and was continuously bubbled with a mixture of $95 \% \mathrm{O}_{2}$ and $5 \% \mathrm{CO}_{2}$ ( $\mathrm{pH} 7.4, \sim 320 \mathrm{mOsm})$. After the incubation, a single brain slice was transferred to a recording chamber placed on the stage of an upright microscope (BX51WI, Olympus, Japan) and submerged in the continuously superfusing medium (1-2 $\mathrm{ml} / \mathrm{min})$. Whole-cell recordings with a high seal resistance ( $>1$ G $\Omega$ before break-in) were obtained from NTS cells with borosilicate glass pipettes (1.5 mm O.D., World Precision Instruments, Sarasota, FL). The electrodes contained (in $\mathrm{mM}$ ): 140 cesium-acetate, $0.1 \mathrm{CaCl}_{2}, 2 \mathrm{MgCl}_{2}, 5$ TEA, 1 EGTA, 10 HEPES, 5 ATP, and $0.1 \%$ biocytin (Sigma-Aldrich, St. Louis, MO, USA) ( $\mathrm{pH}$ 7.3). The resistance of the electrodes filled with this solution ranged from 5 to $12 \mathrm{M} \Omega$. Synaptic currents were recorded in the voltage-clamp mode (Multiclamp 700A, Molecular Devices, Foster City, CA, USA). Currents were filtered at $1-2 \mathrm{kHz}$ and digitized at 2-4 kHz. The amplified signals were displayed, stored, and analyzed on a personal computer with Chart 5/ PowerLab (ADI Japan).

For detecting inward excitatory postsynaptic currents (EPSCs) and outward inhibitory postsynaptic currents (IPSCs) in the same neurons, the membrane potential was clamped first between -60 and $-70 \mathrm{mV}$ and then between 0 and $10 \mathrm{mV}$, respectively. For measuring frequencies of spontaneous postsynaptic currents (sPSCs) in neurons that 
were classified into small or large cells based on biocytinfilled soma, at least $20 \mathrm{~s}$ of continuous sPSC activity was selected at each holding potential. For sPSCs of lower frequency $(<2 \mathrm{~Hz})$, at least $2 \mathrm{~min}$ of current activity was measured. Upward and downward currents of peak amplitudes more than twice the noise levels were sampled using Chart 5 software.

Evoked EPSCs and IPSCs (eEPSCs and eIPSCs) were also recorded and analyzed. Isolated stimuli of 100-200 $\mu \mathrm{s}$ duration were applied at $0.1 \mathrm{~Hz}$ through tungsten bipolar electrodes of tip diameter $20 \mu \mathrm{m}$ and separation $150 \mu \mathrm{m}$. The electrodes were positioned at the dorsomedial part of the tractus solitarius (TS) in coronal slices (Champagnat et al. 1986).

The $\mathrm{GABA}_{\mathrm{A}}$ receptor antagonist bicuculline methiodide $(10 \mu \mathrm{M}$; Sigma-Aldrich) and the non-NMDA glutamate receptor antagonist 6-cyano-7-nitroquinoxaline-2,3-dione disodium (CNQX) (10 $\mu \mathrm{M}$; Sigma-Aldrich) were bathapplied to isolate glutamate- and GABA-mediated activity, respectively.

\section{Reconstruction of labeled neurons}

After recordings, coronal and horizontal slices were fixed in $4 \%$ paraformaldehyde in $0.1 \mathrm{M}$ phosphate buffer for the reconstruction of morphology. After treatment with $0.3 \%$ $\mathrm{H}_{2} \mathrm{O}_{2}$ to suppress endogenous peroxidase and $0.1 \%$ Triton $\mathrm{X}-100$ for better penetration of reagents, the slices were incubated in avidin-biotin-horseradish peroxidase complex (1:300, Vector Laboratories ABC kit, Burlingame, CA, USA) followed by a deaminobenzidine tetrahydrochloride reaction with $\mathrm{H}_{2} \mathrm{O}_{2}$. The slices were then lightly postfixed in $\mathrm{OsO}_{4}$, dehydrated in graded ethanols, and embedded in resin.

Biocytin-filled neurons were photographed with a digital camera and drawn with $40 \times$ objectives, using a camera lucida (Y-IDT Nikon, Japan) attached to a microscope (ECLIPSE, Nikon, Japan). The somal area of neurons was measured based on the photographed soma images using ImageJ (v.1.34n, http://rsb.info.nih.gov/ij/index.html). Manually drawn images of neurons were first scanned into a computer as grayscale images. The neuronal processes (axons and dendrites) of each neuron were then traced using a digital device and converted into bit-mapped digital lines with fixed widths using graphic software (CANVAS11, ACD Systems of America Inc., Miami, FL, USA). The lengths of axons and dendrites of each neuron were measured by selecting digitized line images of each process using built-in commands of CANVAS11. Interrelationships of these morphological parameters were analyzed and represented with OriginPro 8J (LightStone, Tokyo, Japan) by three-dimensionally plotting axonal and dendritic lengths in relation to somal area. These digitized images were also used for line density maps and crossing point analyses.

Digital line drawings $(n=20-30)$ were used to construct two-dimensional (2D) maps of axonal and dendritic arbors and were expressed as 'line density' (Bender et al. 2003; Lübke et al. 2003). Digital line drawings were divided into discrete unit pixels of $1 \mu \mathrm{m}$ side length using built-in commands in Photoshop 5.0.2J (Adobe Systems Inc., San Jose, CA, USA) with a resolution of $1 \mu \mathrm{m} /$ pixel. These pixel representations of axons and dendrites were aligned by soma position with fixed dorsoventral and mediolateral orientations and were then superimposed as stacked drawings. The number of pixels occupied with arbors in $5 \times 5 \mu \mathrm{m}$ grids was calculated by changing a resolution of $1 \mu \mathrm{m} /$ pixel into $5 \mu \mathrm{m} /$ pixel using bilinear interpolation in Photoshop, yielding raw line density images. For averaged representations of the stacked projections, these images were smoothed by a Gaussian blur filter (radius: 5 pixels) in Photoshop. Then, color-coded 2D density maps were constructed using OriginPro 8J.

To determine the ratio of the arbor distributed dorsoventrally within $100 \mu \mathrm{m}$ from the soma to total arbor, arbor fields were divided into 5- $\mu \mathrm{m}$ mediolateral-oriented columns. Arbor length was measured within each column. To describe the differences between axonal domains of small and large cells, the distance between soma and the center of a circumscribed square of contours of 50 and $20 \%$ axonal densities in 2D maps was used.

Overlap of axonal and dendritic arbors provided by pairs of neurons (possible pre- and post-synaptic cells, respectively) was quantified. Since this method would tend to overestimate the true number of contacts, the overlap estimation for neuron pairs was done to show how the probability of interaction changes as somal distance varies, and not for a prediction of actual contacts. For this purpose, we evaluated the overlap of axonal and dendritic arbors of 2D digitized pairs of neurons as a function of distance between the parental somas. Crossing points of axonal and dendritic arbors were counted and averaged at $100-\mu \mathrm{m}$ intervals when a presumed postsynaptic neuronal soma was positioned at four locations just laterally, dorsally, medially and ventrally relative to the fixed presynaptic soma (origin of coordinates). Pairs of 15 possible pre- and post-synaptic cells were randomly selected and the number of crossing points was calculated for each pair.

In situ hybridization histochemistry

For intranuclear localization and density of glutamatergic and GABAergic neuronal somas, in situ hybridization histochemistry was performed using probes for vesicular glutamate transporter 2 (VGLUT2) and glutamic acid decarboxylase 67 (GAD67) as previously described 
(Yoshioka et al. 2006). In brief, cDNA fragments corresponding to base pairs $494-3,866$ of the rat VGLUT2/ DNPI gene (gb: AF271235) and base pairs 488-898 of the rat GAD67 gene (gb: X57573) were amplified with a Taq PCR core kit (Qiagen, Tokyo, Japan). The fragments were subcloned into the pGEM-T Easy vector (Promega, Tokyo, Japan). Using these plasmids as templates, sense and antisense single-strand RNA probes were synthesised with a digoxigenin labeling kit (Roche Diagnostics, Tokyo, Japan). Fresh caudal medulla oblongata that did not undergo fixation procedures was cut in $20 \mu \mathrm{m}$ thick coronal sections on a cryostat. The sections were placed on slides (Superfrost-plus; Fisher Scientific, Pittsburgh, PA, USA), dried for $1 \mathrm{~h}$, and fixed in $4 \%$ paraformaldehyde in $0.1 \mathrm{M}$ PB. After washing in distilled water and prehybridization buffer, the sections were hybridized with each probe.

The background colorization reaction was assessed by periodically observing sections hybridized with each sense probe for up to 1 week at room temperature. No non-specific staining was detected with any of the probes. To identify the boundary of the cNTS, some sections were counterstained with $1 \%$ Neutral Red in distilled water with sodium acetate.

Quantification of signal intensities for GAD67 and VGLUT2 were performed on nine sets of adjacent preparations. Densitometric values within five frames (each area size: $100 \times 100 \mu \mathrm{m}$ ) selected randomly in each subnucleus [as described by our previous study; Yoshioka et al. (2006)] were calculated with ImageJ, and mean densitometric values for each subnucleus were obtained. For background densitometric values per preparation, similar measurements were performed in the dorsal motor nucleus of the vagus $(\mathrm{dmnX})$ located ventrally to the NTS. Finally, mean values for each subnucleus were calibrated to that of area 4 . Differences among subnuclei were tested with the non-parametric Mann-Whitney $U$ test.

\section{Data analysis}

For crossing point analyses on pre- and post-synaptic axodendritic arbors, mean numbers were plotted and fitted by OriginPro $8 \mathrm{~J}$. For quantitative densitometry of in situ hybridization histochemistry, optical densities of reaction products were measured from projected images using ImageJ, and the means and standard errors (SEs) were calculated. Statistical differences among subnuclei were tested with the non-parametric Mann-Whitney $U$ test.

For electrophysiological analyses, statistically significant differences in averaged frequency of sEPSCs and sIPSCs among groups were determined by the non-parametric Mann-Whitney $U$ test, while significant differences in averaged frequency distributions of sEPSCs and sIPSCs among groups were determined by the KolmogorovSmirnov test. Changes in post-stimulus PSC frequency were measured by comparing the event frequencies in $200 \mathrm{~ms}$ bins after the stimulus to that of $1 \mathrm{~s}$ pre-stimulus. At least four consecutive stimuli were averaged to characterize the degree and duration of stimulation-evoked changes in PSC frequency. Paired Student's $t$ tests were used to determine significant differences in frequency of PSCs before and after the TS stimulus. Significance was set at a $P$ value of $<0.01$ or $<0.05$.

\section{Results}

Axon collaterals of small cells are the dominant source of presynaptic elements within the NTS microcircuit

Dimensions of axonal and dendritic arborizations of NTS neurons in relation to somal size were analyzed from 66 reconstructed cells. Smaller cells tended to have a larger dimension of axonal arborizations and smaller arborization of dendrites (Fig. 1d), confirming our previous results (Okada et al. 2006). A size cutoff value of $150 \mu \mathrm{m}^{2}$ in somal area was used, which tended to distinguish neurons having axons with many boutons (probable presynaptic sites) from those without (Yoshioka et al. 2006). Small cells $\left(<150 \mu \mathrm{m}^{2}\right.$ in somal area, $\left.n=36\right)$ possessed prominent axon collaterals (in red) with apparent varicosities within the NTS and dendrites (in blue) of poor arborization (Fig. 1a). Large cells $\left(>150 \mu \mathrm{m}^{2}\right.$ in somal area, $\left.n=30\right)$, on the other hand, had dendrites (blue) of more complex arborization and single projection axons (red) with no ( $n=22 ; 73 \%$ ) or few (1-4 branching points; $n=8$ ) collaterals (Fig. 1b). Axonal lengths were $2,477 \pm 150 \mu \mathrm{m}$ (mean \pm SEs) in small cells and $699 \pm 45 \mu \mathrm{m}$ in large cells. Dendritic lengths were $524 \pm 56 \mu \mathrm{m}$ in small cells and $980 \pm 78 \mu \mathrm{m}$ in large cells. The axonal and dendritic lengths were inversely and linearly correlated with somal size (Fig. 1d, correlation coefficients: $r=0.64$ : axon/ soma; $r=0.63$ : dendrite/soma). These results suggest that axon collaterals of small cells are the dominant source of presynaptic elements of the NTS microcircuit.

Axonal and dendritic arbors radiate in a spatially even manner

The microcircuit of the NTS thus seems to be comprised mainly of putative presynaptic axons of small cells and postsynaptic dendrites of NTS cells. Functional connections require overlap of presynaptic axons and postsynaptic dendrites. To quantify this overlap, it would be necessary to first characterize the dimensions of axonal and dendritic arbors. Axons (red) and dendrites (blue) of 29 
A

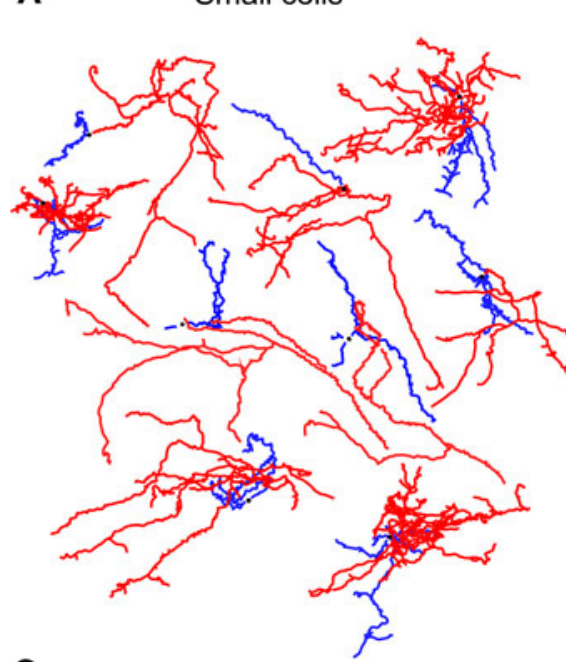

C

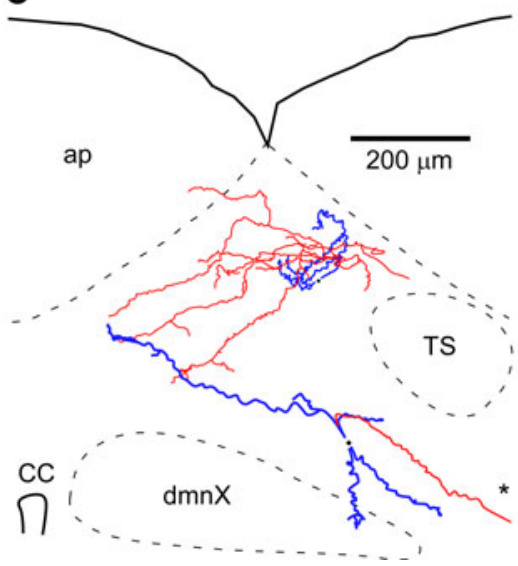

Fig. 1 Features and dimensions of axonal and dendritic arbors. a, b Examples of reconstructed small (a) and large (b) cells with orientations, depicted as digitized images with somas (black dot), axons (red) and dendrites (blue) are represented. c Schematic representation of a pair of a small (upper) and a large (lower) cell in their distinct locations. An asterisk indicates the main axon exiting from the nuclear boundary (truncated). d Three-dimensional plot of axonal length (red squares) and dendritic length (blue squares) in relation to somal size (in area) of 66 NTS neurons (yellow spheres

reconstructed small cells and dendrites (blue) of 23 large cells from coronal slices, together with dendrites of 13 small and 14 large cells from horizontal slices, were aligned by soma position and two-dimensionally superimposed (Fig. 2a1-c1 and Supplementary Fig. 1). To characterize the anatomical organizations of process arbors, we quantified two-dimensionally projected process arbors in terms of line density, averaged across many process arbors, and constructed 2D line density maps for each process (Fig. 2a2-c2 and Supplementary Fig. 1). Each process density distributed binomially with a peak located near the soma position, except for large cell axons, which are distributed in a spatially uneven and non-concentric manner.
B Large cells
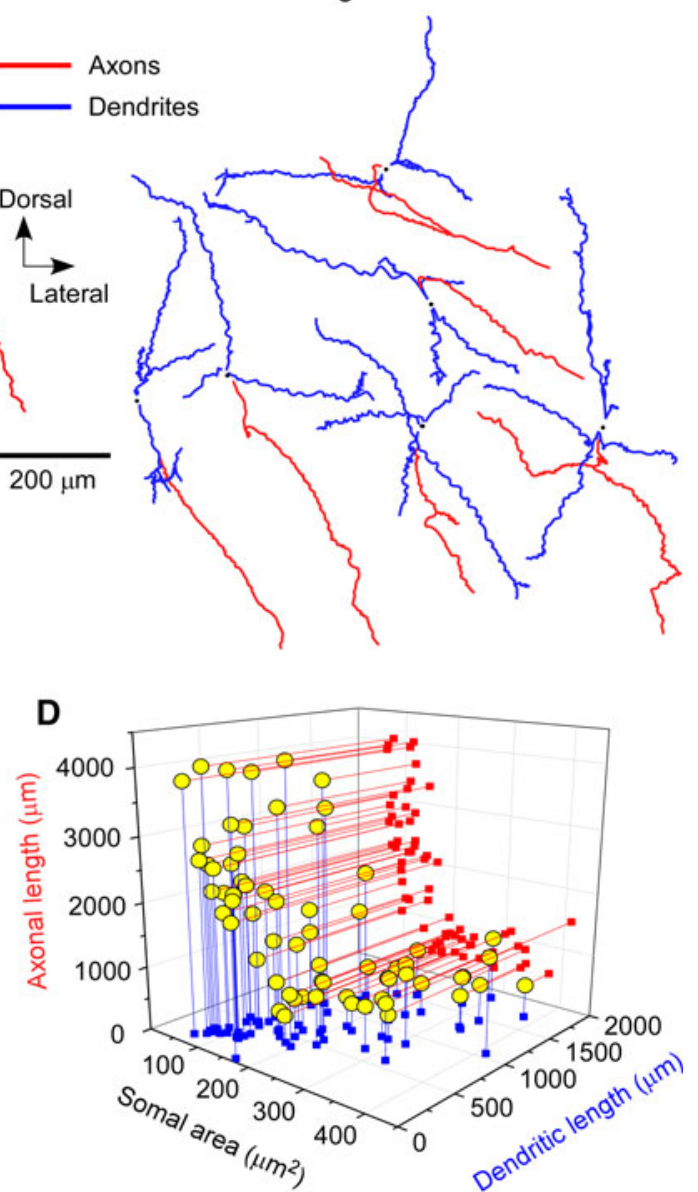

indicate the three- dimensional values for single cells; thin lines projecting to zero values are also included for better visualization of the relative positions of the spheres). Note that smaller cells have more extensive axon arbors and are thus likely to play a role as presynaptic neurons in the cNTS. A value of $150 \mu \mathrm{m}^{2}$ in somal area was used to distinguish between small and large cells. ap The area postrema, $C C$ central canal, $d m n X$ the dorsal motor nucleus of the vagus nerve, $T S$ the tractus solitarius

About $60 \%$ of small cell axons were distributed dorsoventrally within $100 \mu \mathrm{m}$ from the soma. The densities were decreased dorsoventrally to $50 \%$ at $\sim 110 \mu \mathrm{m}$ and to $20 \%$ at $\sim 200 \mu \mathrm{m}$ from soma (Fig. 2a2). About $70 \%$ of small cell dendrites were distributed within $100 \mu \mathrm{m}$ from soma, and the densities were decreased to $50 \%$ at $\sim 60 \mu \mathrm{m}$ and to $20 \%$ at $\sim 150 \mu \mathrm{m}$ from soma (Fig. 2b2). Likewise, about $65 \%$ of large cell dendrites were distributed dorsoventrally within $100 \mu \mathrm{m}$ from soma, and the densities were decreased to $50 \%$ at $\sim 70 \mu \mathrm{m}$, and to $20 \%$ at $\sim 170 \mu \mathrm{m}$ from soma (Fig. 2c2). Furthermore, the present analysis of reconstructed cells confirmed the spatially even distribution of process arbors observed in horizontal slices (Supplementary Fig. 1; Zhang et al. 1995). The distances 
Fig. 2 Geometric maps of axonal and dendritic arbors of cNTS neurons. a1-c1 Each reconstructed cell process was aligned by soma position and digitally superimposed; axons (a1 in red), dendrite arbors of 29 small cells (b1 in blue), and dendrites of 23 large cells (c1, in blue). a2-c2 Twodimensional maps of axonal (a2) and dendritic (b2 small cells; c2 large cells) arbors expressed as line density based on the digitized images (a1-c1) after a Gaussian smoothing. For each panel, $100 \%$ refers to maximum line density. Contours (black lines) of 20 and $50 \%$ density were shown
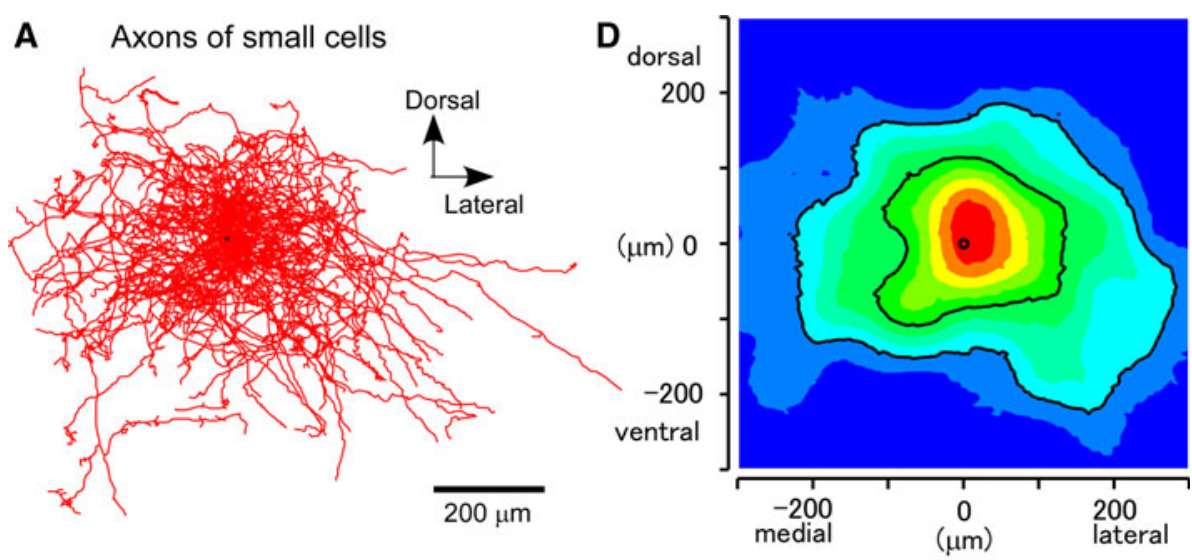

B
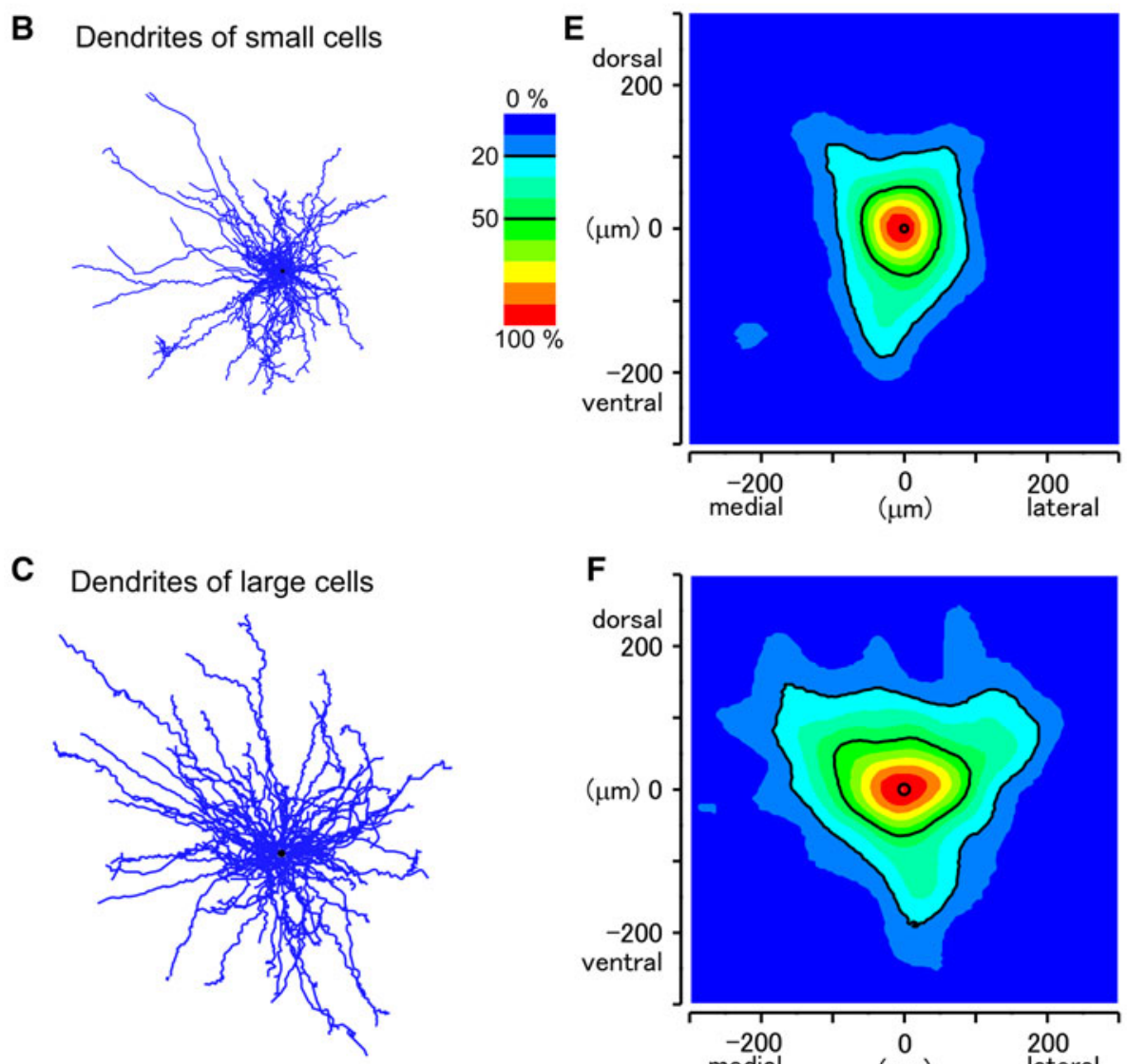

between soma and the center of the circumscribed square of contours for 50 and $20 \%$ axonal density in 2D maps were smaller in small cells (c. 9 and $30 \mu \mathrm{m}$ ) as compared to large cells (c. 130 and $240 \mu \mathrm{m}$ ). These results suggest that axonal and dendritic arbors of small cells and the latter of large cells radiate in a spatially even manner.

\section{Geometric relation of axons and dendrites}

Next, two-dimensionally projected axons and dendrites were used to quantify the possible overlap in relation to distance between their parent somas. Superimposed axons of small neurons seemed to extend mostly over superimposed dendrites of small and large neurons when both somas were centered (Fig. 3a, b). We selected pairs of putative presynaptic axonal arbors and postsynaptic dendrites from 15 small and large reconstructed cells, respectively, and counted crossing points of axons and dendrites in relation to distances between their parent somas (Fig. 3c). Averages of crossing point values against soma distances could be fitted by Gaussian curves (Fig. 3d). Numbers of crossings decreased to $50 \%$ at 
Fig. 3 Overlap of axon and dendrite arbors. a, b Density overlap between superimposed axonal (putatively presynaptic) and dendritic (postsynaptic) arbors of small (a) and large (b) cells, aligned by soma position with dotted concentric circles separated by $100 \mu \mathrm{m}$. Note the wider dimension of high-density overlap for large postsynaptic cells. c Point-topoint overlap (magnified in a circle) between axons and dendrites from pairs of pre- and postsynaptic cells in relation to their somal distance. In this case, three crossings can be identified. d Average number of crossing points as a function of distance between pre- and postsynaptic cells. Vertical bars represent standard errors. The plots of postsynaptic small cells (squares) and large cells (circles) were fitted by Gaussian curves (black lines)
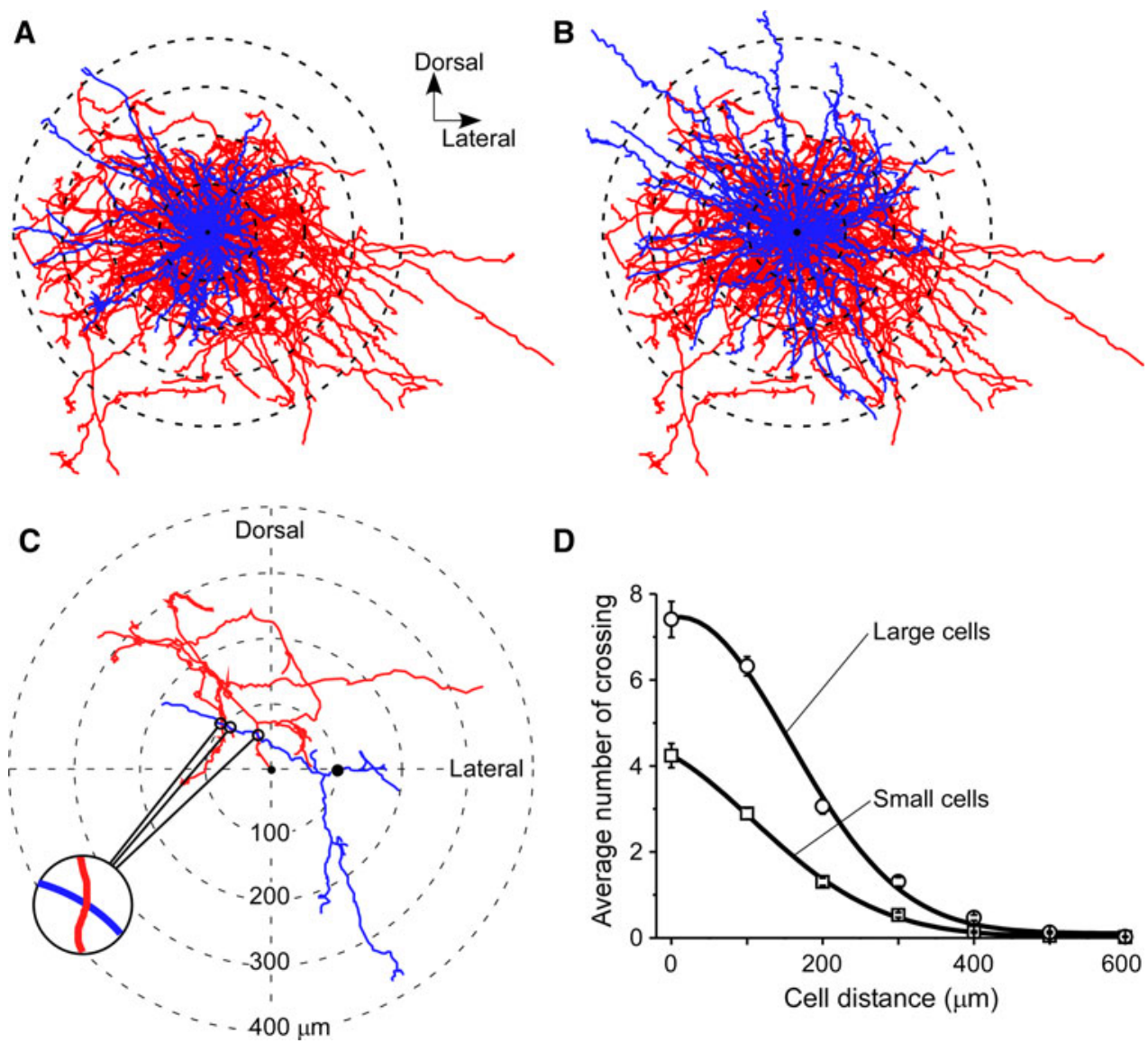

D

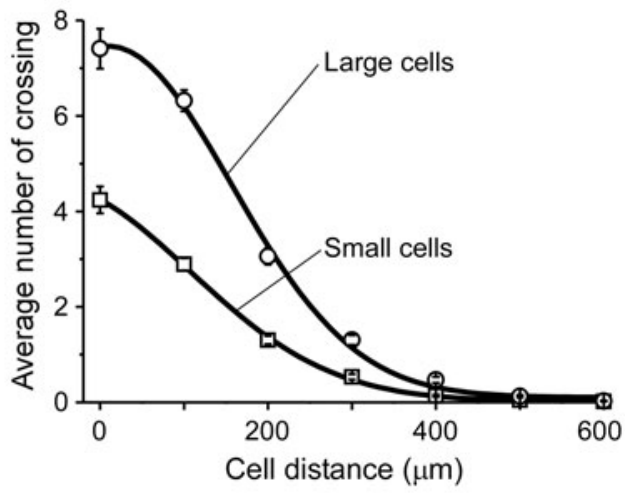

$\sim 150 \mu \mathrm{m}$ between small cells, and at $\sim 200 \mu \mathrm{m}$ between small and large cells. A common feature was that axon and dendrite overlap exponentially increased as parent somas became closer. Since actual synapses need close threedimensional appositions between axons and dendrites, the probability of occurrence of functional synapses would drastically decrease when the distances between parent somas exceed $200 \mu \mathrm{m}$ in both cases.

GABAergic cells are distributed mostly in the ventral cNTS: anatomical segregation of excitability

Uneven distribution of GABAergic cells was semi-quantitatively analyzed in four subregions (areas 1-4) of the cNTS (Fig. 4f) (Yoshioka et al. 2006; Okada et al. 2008). Signals for GAD67, a GABAergic neuronal marker, were weak in the dorsal regions (Fig. 4c, d), while signals of VGLUT2, a glutamatergic marker, were evenly strong throughout the cNTS (Fig. 4a, b). Since area 4 has been reported to have the highest neuronal density and contain nearly equal densities of glutamatergic and GABAergic cells (Yoshioka et al. 2006; Okada et al. 2008), densitometric values for GAD67 and VGLUT2 in each area were calibrated to those in area 4 (Fig. 4e). Signals for VGLUT2 were 96\% (area 1), 99\% (area 2), and 94\% (area 3) of area 4, while those for GAD67 were 30, 31, and $100 \%$, respectively. Areas 1 and 2, which correspond to a dorsal subregion of the cNTS, contained significantly less signal (30\% in area 1 and 31\% in area 2) for GAD67 than not only that of ventral regions but also for that for VGLUT2. The width of dorsal and ventral laminae was, interestingly, less than $200 \mu \mathrm{m}$, a range of distance between parent somas that could produce axo-dendritic overlap.

Synaptic activity patterns differed dorsoventrally in the cNTS: functional segregation of excitability

Spontaneous postsynaptic activity patterns were analyzed to examine whether the anatomical differentiation of potential excitability reflects a difference in functional dynamics. We have previously reported a differentiation of spontaneous postsynaptic current patterns according to soma size (Kawai and Senba 1999, 2000; Yoshioka et al. 2006). In our present study, sEPSCs and sIPSCs were analyzed according to not only cell size but also with regard to soma location within the cNTS.

Since there are few (less than $\sim 5 \%$ ) large cells in the dorsal region (Yoshioka et al. 2006), neurons were divided into the following three groups: (1) dorsal small cells 
Fig. 4 Somal distribution of glutamatergic and GABAergic neurons according to VGLUT2 and GAD67 mRNA expression profiles. a, b In situ hybridization histochemistry using probes for VGLUT2 (a) and GAD67 (b) were performed on a set of adjacent preparations. c, d Superposition of positive signals (black) in six preparations for VGLUT2 (c) and GAD67 (d). Note that the same expression patterns of VGLUT2 and GAD67 mRNA were observed at the level of the area postrema. e Relative densitometric values for VGLUT2 ( $n=9$, open column) and GAD67 $(n=9$, solid column) mRNA in each subnucleus [as proposed by our previous study; Yoshioka, et al., (2006)] were calculated. Data are mean \pm SEs. Significant differences were determined by the Mann-Whitney $U$ test. Asterisks denote $P$ value of $<0.01$. f Five frames of $100 \times 100 \mu \mathrm{m}$ sections selected randomly in each area were analyzed for optical densitometric measurements of reaction product with ImageJ. ap the area postrema, $C C$ central canal, $d m n X$ the dorsal motor nucleus of the vagus nerve, $T S$ the tractus solitarius
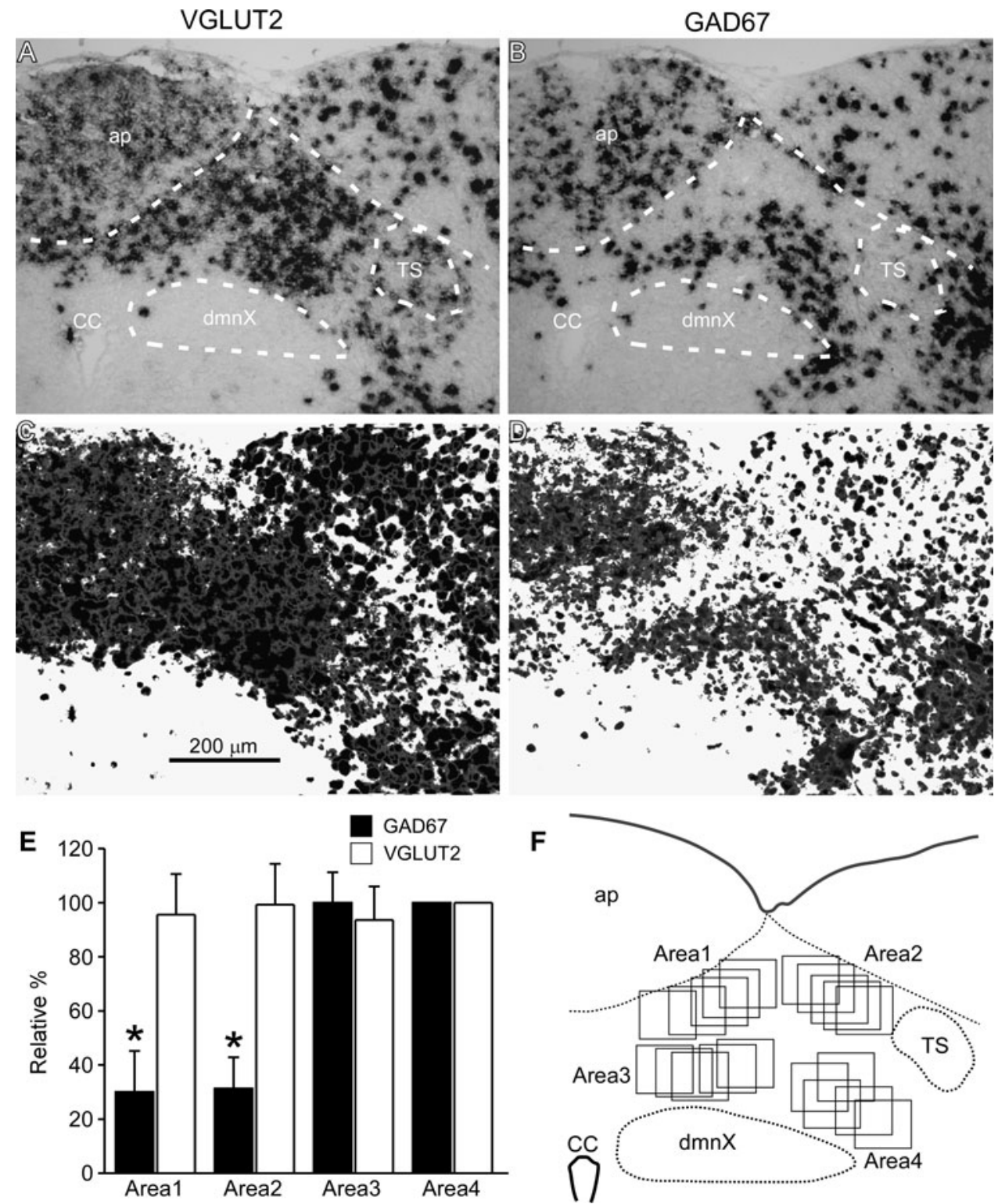

(Ds; $n=30$ ), (2) ventral small cells (Vs; $n=32$ ), and (3) ventral large cells $(\mathrm{Vl} ; n=24)$. The results are shown in Fig. 5 and Table 1. Inward EPSCs and outward IPSCs were completely blocked by CNQX $(10 \mu \mathrm{M})$ and bicuculline $(10 \mu \mathrm{M})$, respectively (data not shown), confirming that they represented glutamatergic and GABAergic PSCs, respectively. Patterns of sPSCs were statistically different not only according to somal size but also somal location according to Mann-Whitney $U$ tests. In small cells, significantly higher frequencies of sEPSCs and lower frequencies of sIPSCs were evident in Ds than Vs (sEPSCs: $P<0.01$, sIPSCs: $P<0.05$ ) (Table 1 ). In the ventral region, statistically significant differences were noted between Vs and $\mathrm{Vl}$ in the frequency of sEPSCs $(P<0.01)$ and sIPSCs $(P<0.01)$ (Table 1$)$. The KolmogorovSmirnov test on neurons having higher PSC frequency in each group revealed statistically significant differences $(P<0.01)$ among groups across size and location (Fig. 5b, d).

Laminar differences in synaptic dynamics

In addition to spontaneous postsynaptic current patterns, we next analyzed evoked postsynaptic current patterns to address the possible laminar differences in synaptic dynamics.

TS stimulation evoked fast monosynaptic PSCs $(<6 \mathrm{~ms}$ latency) that possibly derived from primary afferents or nearby cells, and polysynaptic multiple ones that could last for up to several seconds (Fortin and Champagnat 1993; Smith et al. 1998; Kawai and Senba 1999). Four patterns of evoked PSCs were recorded from Ds $(n=8)$, Vs $(n=6)$ 
A

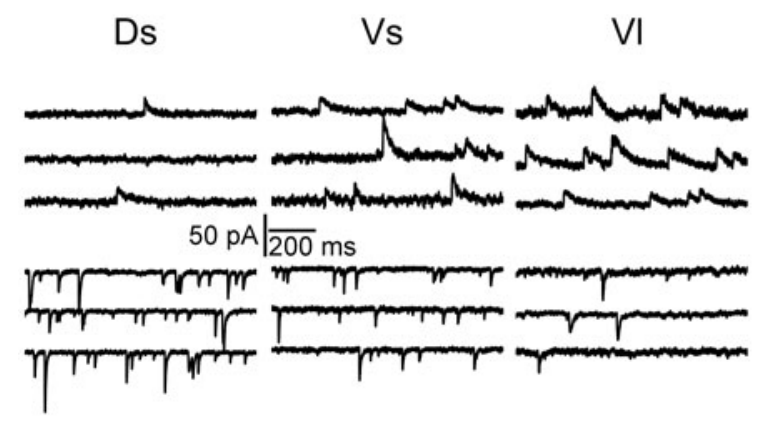

C

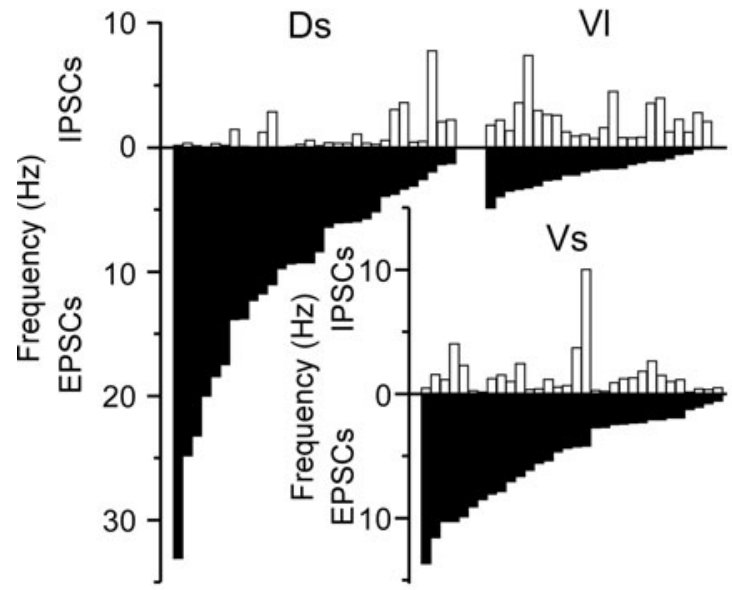

B

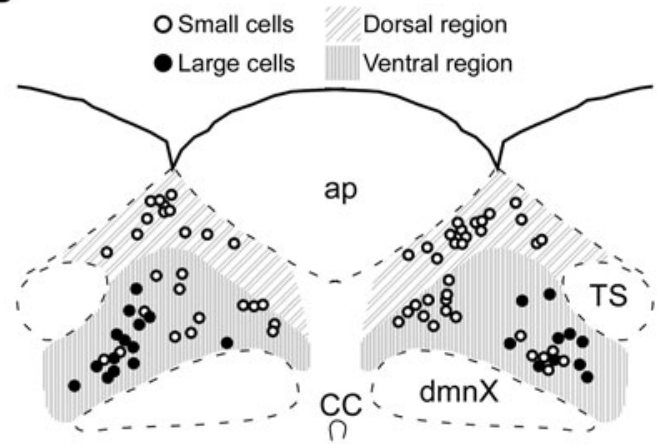

D
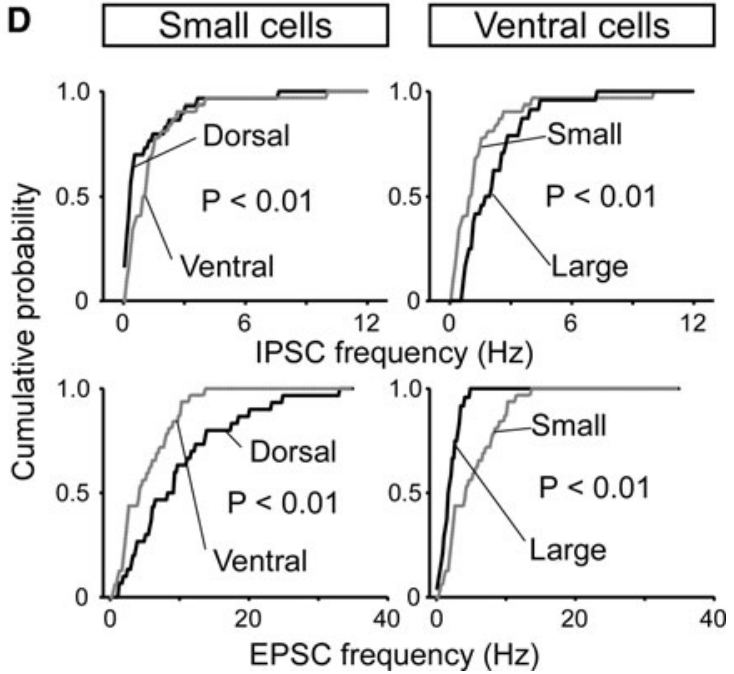

sIPSCs from single Ds (upper left), Vs (upper right), and Vl (lower) cells. Open and solid columns arranged from left to right according to the sEPSC frequencies show frequencies of sEPSCs and sIPSCs recorded from single cells, respectively. $\mathbf{d}$ Cumulative distributions of EPSC and IPSC frequency of Ds, Vs, and Vl. Frequency plots of dorsal small and ventral large (black) and ventral small (gray) cells were constructed and the Kolmogorov-Smirnov test was used to determine significant differences between cumulative distributions. The frequency distributions were significantly different $(P<0.01)$ between dorsal and ventral small cells and between ventral small and large cells

Table 1 Frequencies of sEPSCs and sIPSCs in Ds, Vs and Vl

\begin{tabular}{llllll}
\hline & Ds $(n=30)$ & Vs $(n=32)$ & Vl $(n=24)$ & Statistical analysis \\
\cline { 3 - 6 } & & & Ds versus Vs & Vs versus V1 \\
\hline sEPSC frequency $(\mathrm{Hz})$ & $10 \pm 1.4(1.3-33)$ & $5.1 \pm 0.6(0.6-14)$ & $2.0 \pm 0.3(0-4.9)$ & $P<0.01$ & $P<0.01$ \\
sIPSC frequency (Hz) & $1.0 \pm 1.6(0-7.7)$ & $1.4 \pm 1.8(0.1-10)$ & $2.2 \pm 0.3(0.7-7.3)$ & $P<0.05$ & $P<0.01$ \\
\hline
\end{tabular}

Values are mean \pm SEs, with the ranges of events analyzed in parentheses. Significant differences among groups were determined by the MannWhitney $U$ test

$D s$ dorsal small cells, $V s$ ventral small cells, $V l$ ventral large cells

and $\mathrm{Vl}(n=11)$ by TS single stimulations: eEPSC, eEPSC and eIPSC, eIPSC, and non-response. In Ds, eEPSCs were the most frequently observed pattern $(n=5 ; 63 \%)$, followed by non-response $(n=2)$, eEPSCs and eIPSCs $(n=1)$, and eIPSCs $(n=1)$. In Vs, eEPSCs and eIPSCs were the most frequently observed pattern $(n=4 ; 67 \%)$ followed by eEPSCs $(n=2)$. In Vl, eEPSCs and eIPSCs were the most frequently observed pattern $(n=7 ; 64 \%)$, 
followed by eEPSCs $(n=3)$ and non-response $(n=1)$. The most prevalent patterns of ePSCs in Ds, Vs, and Vl were similar to those for the respective sPSCs.

Single TS stimulation evoked not only monosynaptic but also multiple responses (Fig. 6a, b) as shown in previous studies (Fortin and Champagnat 1993; Kawai and Senba 1996; Smith et al. 1998). Barrages of either EPSCs or IPSCs were observed in one small and nine large neuron(s) (Fig. 6). Multiple responses after TS stimulation were considered as a barrage of PSCs when statistically significant differences between PSC frequency before and after TS stimulation were detected by paired Student's $t$ tests $(P<0.05)$. A barrage of EPSCs, lasting from 200 to $1,400 \mathrm{~ms}$, was observed in one Ds and four $\mathrm{Vl}$. A barrage of IPSCs, lasting from 200 to $1,200 \mathrm{~ms}$, was observed in five Vl. Barrages of both EPSCs and IPSCs evoked by TS stimulation were abolished by $10 \mu \mathrm{M}$ CNQX (Fig. 7), suggesting that local glutamatergic transmission mediated the lasting activity. Under our conditions, there is a possibility that the EPSCs contain a minor NMDA component that seems to range around the noise level. However, this component would be expected to increase under conditions with glycine and no magnesium (Aylwin et al. 1997).

\section{Discussion}

Anatomical and functional laminar organization of the cNTS was described in terms of excitability differentiation as characterized by an architecture and dynamics of cell assembly that seems to be specific to this nucleus.

The anatomical basis for our hypothesis lies in an uneven distribution of inhibitory cells in this nucleus and the resultant assembly of specific neuronal connectivity as predicted by Peters' rule. This rule states that neurons interconnect in proportion to the neuropil contribution from their dendrites and axonal varicosities (Braitenberg and Schuz 1998), and has been substantiated by theoretical and realistic neurogeometry (Shepherd et al. 2005; Stepanyants and Chklovskii 2005). The rule requires an assumption that axonal varicosities be homogeneously distributed and roughly similar for different types of axons, and therefore, presumably cannot be applied universally for every system. However, on average, connectivities between various types of cerebral cortical neurons, as well as between cerebellar Purkinje cells, are reported to follow Peters' rule (Shepherd et al. 2005; Watt et al. 2009). Accordingly, it is likely to be the case for NTS neuronal connectivity, given that the requisite assumptions seemed to be generally satisfied in our present study. In conclusion, the cNTS consists of two distinct laminas of cell assembly: a dorsal cell assembly interconnected exclusively by excitatory synapses and a ventral one interconnected by mixed excitatory and inhibitory synapses.

Functional lamination was suggested by the presence of differentiated synaptic dynamics in terms of spontaneous and evoked postsynaptic current patterns. Given that we were able to distinguish location-specific patterns of postsynaptic activity, we believe that functional cell assemblies and synapses were likely preserved in slice preparations used in the present study. The dorsal cNTS region contained cell assemblies of higher excitability in terms of sEPSC frequency. Mixed inhibitory and excitatory postsynaptic activities were more evident with reverberation lasting several seconds in neurons located in the ventral subregion of the cNTS.

Thus, the anatomical and functional quantification of connectivities would seem to be sufficiently correlated to justify a laminar organization of excitability.

\section{Laminar organization}

Since the time of Ramon y Cajal, the NTS has been considered a visceral sensory nucleus of homogeneous cell architecture (Ramon y Cajal 1995). Although some subnuclear classification has been described in the literature, these studies were based mostly on Nissl-staining and do not refer to laminar organization (Kalia and Sullivan 1982). Unlike the spinal cord dorsal horn, in which specific neuronal types with laminar organization have been understood for some time, the cytoarchitecture of the NTS (based on Golgi staining or intracellular labeling preparations) has been revealed only recently (Whitehead 1988; Kawai and Senba 1996). Judging from our reconstructed materials thus far obtained, there seem to be no major morphological differences between ventral and dorsal small cells. However, there is a possibility that sampling larger numbers could reveal a novel type of small cell. The results of these studies seem to confirm a homogeneous neuronal organization, and this evidence was partly substantiated by electrophysiological measures (Zhou and Poon 2000). Despite these investigations, the intrinsic neuronal connectivity (i.e., the microcircuit) is still poorly understood, and has not been studied in terms of laminar organization. However, potential laminar organization of the cNTS has in fact been suggested from other points of view, including studies of afferent and efferent labeling with neuronal tracers (Herbert et al. 1990; Altschuler et al. 1991; van der Kooy et al. 1984) and in vivo recordings of response patterns (McCann and Rogers 1992).

The ventral region, corresponding to the medial and commissural subnuclei, and the dorsal region, being comprised of the dorsolateral subnucleus and subpostremal zone, contains distinctive neuronal groups projecting to differential parabrachial subregions (Herbert et al. 1990). 
Fig. 6 Spatial differentiation of evoked postsynaptic activities. a, b Three consecutive traces showing excitatory (a) and inhibitory (b) postsynaptic currents following single TS stimulations obtained from two different cells. Arrowheads indicate TS stimulation.

Stimulation artifacts were truncated for clarity. Evoked inward EPSCs and outward IPSCs were recorded from each cell at holding potentials of $0-10 \mathrm{mV}$ and -60 to $-70 \mathrm{mV}$, respectively. c, d Mean frequency of EPSCs for four consecutive trials (c) and IPSCs for five consecutive trials (d) relative to time of stimulation (200 ms bins, mean \pm SEs). Asterisks above the bars indicate significantly increased frequency $(P<0.05$, paired Student's $t$ test) when compared with the averaged pre-stimulus frequency. e Schematic representation of location of recorded small (circles) and large (stars) neurons in the dorsal (slashed area) and ventral (striped area) regions. In addition, evoked postsynaptic activities of eEPSCs alone (open), eIPSCs with and without a precedent unitary eEPSC (solid), and barrage of PSCs (enclosed square) are shown. Ds dorsal small cells, $V s$ ventral small cells, $V l$ ventral large cells, ap the area postrema, $C C$ central canal, $d m n X$ the dorsal motor nucleus of the vagus nerve, $T S$ the tractus solitarius
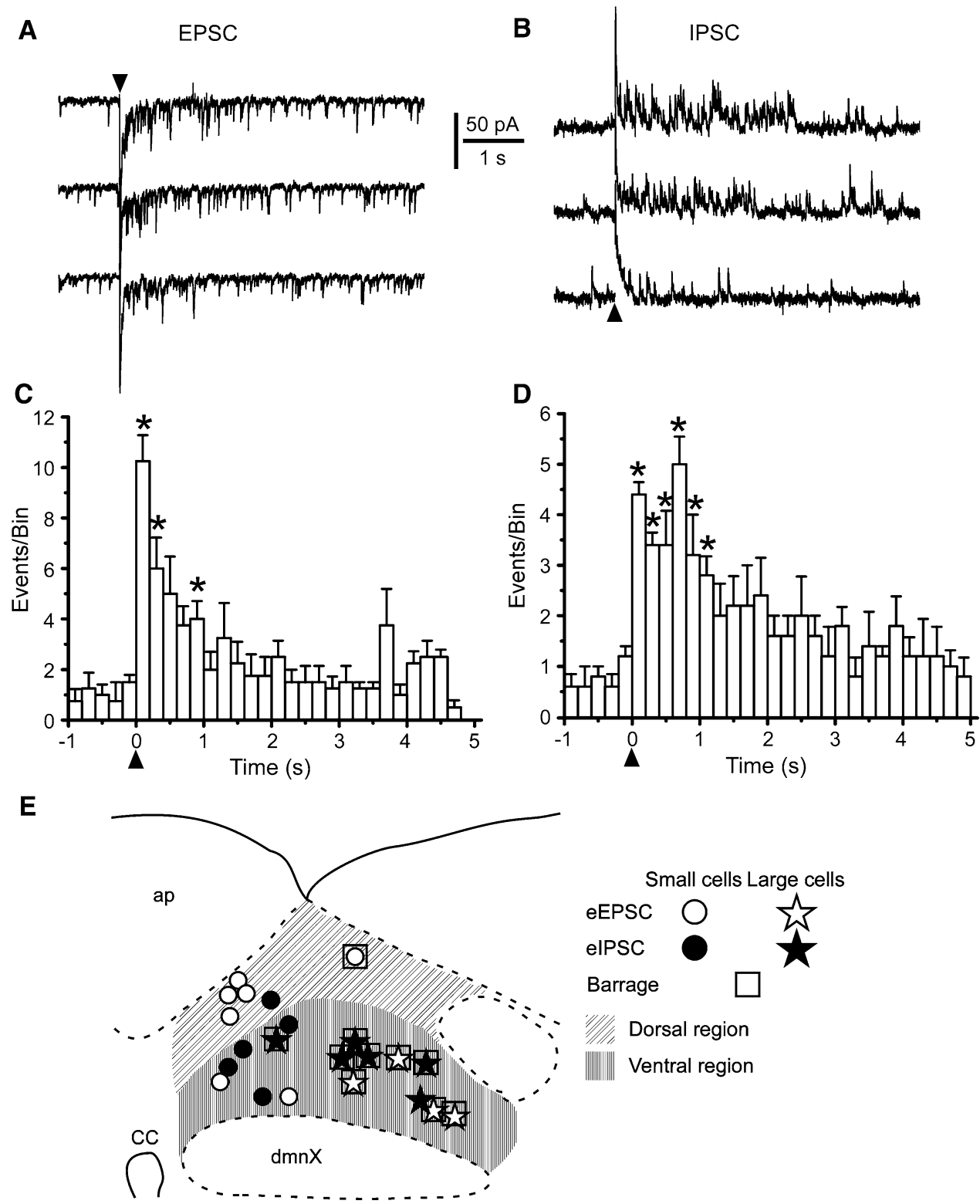

The topological efferent projection system from the NTS to the parabrachial nucleus is likely to correspond to viscerotopic representations of the peripheral afferent system (Altschuler et al. 1989, 1991). As to the central afferent system, there also seems to be a laminar distribution according to different origins (van der Kooy et al. 1984). In addition to topological representation of projections, the dorsal region seems to receive much denser innervations of peripheral visceral afferents than the ventral (Aicher et al. 1999; Chan et al. 2000). Accordingly, the neuronal activation marker c-Fos is more likely to be expressed in dorsal NTS cells in response to baroreceptor activation (Weston et al. 2003). Qualitative and quantitative differences in cell assembly representations of efferent and afferent projection systems could correspond to the spatial organization of excitability indicated by the present study.

McCann and Rogers (1992) described excited and inhibited cells in response to stomach distension along various depths from the dorsal surface of the brainstem, and reported the presence of the former in the dorsal NTS and the latter in the ventral NTS and dmnX. These results obtained from in vivo recordings are fairly consistent with our present study using slice preparations.

The NTS may be a part of the dorsal vagal complex, which additionally includes a parasympathetic preganglionic neuronal group in the $\mathrm{dmnX}$. In this case, the dorsal vagal complex would consist of at least three layers. The $\mathrm{dmnX}$ is almost devoid of glutamatergic and GABAergic 


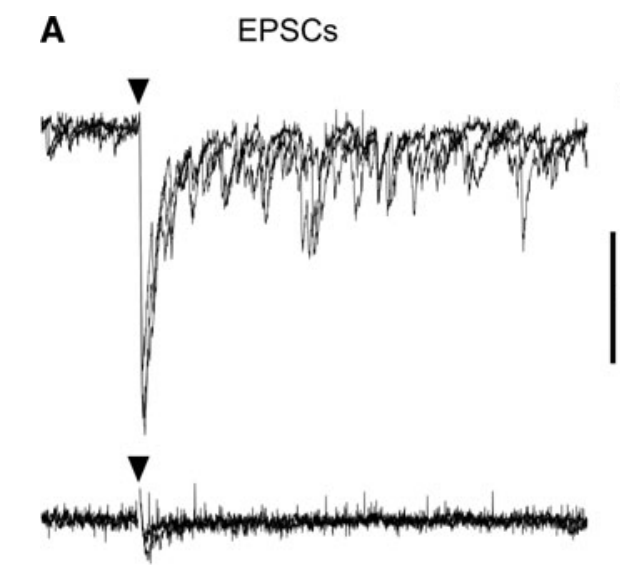

Fig. 7 Glutamatergic transmission was required for the prolonged barrage of both EPSCs and IPSCs. a, b Three superimposed consecutive sweeps (1 s) recorded from one cell with a barrage of EPSCs (a) and another cell with a barrage of IPSCs (b) under control conditions (upper) and in the presence of $10 \mu \mathrm{M}$ CNQX, a non-

cells, and consists primarily of cholinergic cell groups. Dendrites of dmnX cells extend into ventral regions of the cNTS, in which GABAergic cells aggregate (Fig. 4) (Shapiro and Miselis 1985). It is likely that dmnX cells are under an intense GABAergic influence just like ventral NTS cells, and this is supported by both in vivo and in vitro electrophysiological studies (McCann and Rogers 1992; Zhang et al. 1998; Davis et al. 2004). Thus, a gradient of excitability seems to be formed along a dorsoventral axis within the dorsal vagal complex as a whole.

\section{Analogies to cerebral cortical microcircuitry}

In addition to the fundamental laminar and columnar organization of the cerebral neocortex, the most spectacular aspect of cortical microcircuit architecture is likely to consist in the fact that most cortical connections are local and excitatory (Douglas and Martin 2007). Intriguing properties of cortical microcircuits have also arisen from quantitative anatomical data (Binzegger et al. 2004). It is likely that local cortical synapses dominate over extrinsic afferents from the thalamus and other cortical areas. For example, in the visual cortex, synapses from the lateral geniculate nucleus constitute less than $10 \%$ of excitatory synapses. The largest fraction of synapses in the microcircuitry is excitatory connections formed between pyramidal neurons of the superficial layers. If there indeed exist common properties of microcircuits among different brain regions, it would be of great interest to determine whether similar computations can be performed in distinct microcircuits.

There is little quantitative anatomical data concerning the weight of extrinsic afferent synapses in the cNTS. An electron microscopic study determined that $11 \%$ of all cNTS synapses were peripheral afferent synapses (Chiba

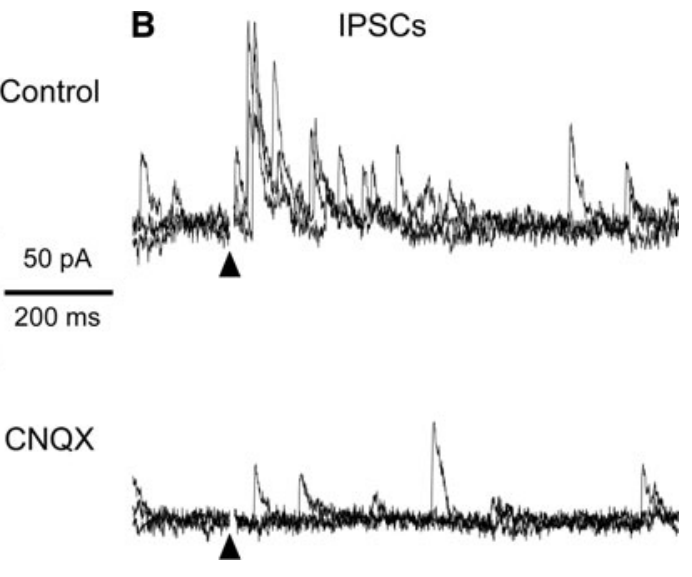

NMDA receptor antagonist (lower). Arrowheads indicate TS stimulation. Stimulation artifacts were truncated for clarity. Evoked inward EPSCs and outward IPSCs were recorded from each cell at holding potentials of $0-10 \mathrm{mV}$ and -60 to $-70 \mathrm{mV}$, respectively

and Kato 1978). Immunohistochemistry using antibodies against VGLUT1\&2 (as a glutamatergic presynaptic axon marker) has revealed few differences in staining in the cNTS after peripheral deafferentation, confirming a dominance of intrinsic synapses (Lachamp et al. 2006; our observation). Thus, there may be some similarity between the cerebral cortex and NTS microcircuits with regard to extrinsic afferent levels.

There also seems to be some commonalities between the cerebral cortex and cNTS in terms of gradients of organizational properties along a perpendicular (dorsoventral) axis. Pyramidal cells in the cortical superficial layers have a smaller somatic size and stronger excitatory connections as compared to deeper pyramidal cells. The cNTS neurons in the dorsal subregion have smaller somas versus the deeper projection cells, including dmnX neurons. Also, the former forms stronger excitatory local connections (due to an absence of nearby GABAergic cells) than the latter (due to a lack of axon collaterals). Finally, GABAergic neurons are arranged more compactly in the vicinity of projection cells with fewer axon collaterals in both brain areas. More detailed quantitative comparisons will be necessary to further understand the specific computations carried out in these microcircuits.

Functional implications

Nearly $70 \%$ of a superficial pyramidal cell's excitatory input is derived from other cells of its own type, making it more likely to give rise to recurrent connections between superficial pyramidal cells (Binzegger et al. 2004; Douglas and Martin 2007). This seems to be the case with dorsal cNTS neurons, given that the dorsal cNTS contains mostly excitatory small cells of a similar type. What type of 
synaptic activity could such recurrent connections give rise to? It is likely that persistent or reverberating recurrent activities could be produced by recurrent excitatory connections in the cerebral cortex (Sanchez-Vives and McCormick 2000; Yuste et al. 2005) and the NTS (Champagnat et al. 1986; Kawai and Senba 1996; Smith et al. 1998; Davis et al. 2004). However, it has been difficult to investigate such reverberation of synaptic activity in vitro, possibly due to the enormous complexity of microcircuits. Cultured hippocampal neurons have been used to address this question. However, in this preparation the recurrent synaptic connections were far from native (Lau and Bi 2005). The NTS might provide an experimental substrate in which relationships between native microcircuit organization and reverberatory activity both in vitro and in vivo can be investigated for better understanding of signal computation.

It has recently been proposed that positive feedback plays a crucial role in cortical computation by providing gain for the active selection and re-combination of relatively small afferent signals (Douglas and Martin 2007). Such non-linear signal processing might be implicated in the NTS microcircuit, and this factor should be investigated in future experiments.

Acknowledgments This study was supported by grants from the Showa Kaikan Foundation to Jikei University School of Medicine.

Open Access This article is distributed under the terms of the Creative Commons Attribution Noncommercial License which permits any noncommercial use, distribution, and reproduction in any medium, provided the original author(s) and source are credited.

\section{References}

Aicher SA, Sharma S, Pickel VM (1999) $N$-methyl-D-aspartate receptors are present in vagal afferents and their dendritic targets in the nucleus tractus solitarius. Neuroscience 91:119-132. doi:10.1016/S0006-8993(97)00637-9

Altschuler SM, Bao X, Bieger D, Hopkins DA, Miselis RR (1989) Viscerotopic representation of the upper alimentary tract in the rat: sensory ganglia and nuclei of the solitary and spinal trigeminal tracts. J Comp Neurol 283:248-268. doi:10.1002/cne.902830207

Altschuler SM, Ferenci DA, Lynn RB, Miselis RR (1991) Representation of the cecum in the lateral dorsal motor nucleus of the vagus nerve and commissural subnucleus of the nucleus tractus solitarii in rat. J Comp Neurol 304:261-274. doi:10.1002/cne. 903040209

Aylwin ML, Horowitz JM, Bonham AC (1997) NMDA receptors contribute to primary visceral afferent transmission in the nucleus of the solitary tract. J Neurophysiol 77:2539-2548

Bender KJ, Rangel J, Feldman DE (2003) Development of columnar topography in the excitatory layer 4 to layer $2 / 3$ projection in rat barrel cortex. J Neurosci 23:8759-8770

Binzegger T, Douglas RJ, Martin KA (2004) A quantitative map of the circuit of cat primary visual cortex. J Neurosci 24:84418453. doi:10.1523/JNEUROSCI.1400-04.2004
Braitenberg V, Schuz A (1998) CORTEX: statistics and geometry of neuronal connectivity, 2nd edn. Springer, Berlin

Champagnat J, Siggins GR, Koda LY, Denavit-Saubié M (1985) Synaptic responses of neurons of the nucleus tractus solitarius in vitro. Brain Res 325:49-56. doi:10.1016/0006-8993(85) 90301-4

Champagnat J, Denavit-Saubié M, Grant K, Shen KF (1986) Organization of synaptic transmission in the mammalian solitary complex, studied in vitro. J Physiol 381:551-573

Chan RKW, Jarvina EV, Sawchenko PE (2000) Effects of selective sinoaortic denervations on phenylephrine-induced activational responses in the nucleus of the solitary tract. Neuroscience 101:165-178. doi:10.1016/S0306-4522(00)00332-8

Chiba T, Kato M (1978) Synaptic structures and quantification of catecholaminergic axons in the nucleus tractus solitarius of the rat: possible modulatory roles of catecholamines in baroreceptor reflexes. Brain Res 151:323-338. doi:10.1016/0006-8993(78) 90888-0

Davis SF, Derbenev AV, Williams KW, Glatzer NR, Smith BN (2004) Excitatory and inhibitory local circuit input to the rat dorsal motor nucleus of the vagus originating from the nucleus tractus soitarius. Brain Res 1017:208-217. doi:10.1016/j.brainres. 2004.05.049

Douglas RJ, Martin KAC (2007) Recurrent neuronal circuits in the neocortex. Curr Biol 17:R496-R500. doi:10.1016/j.cub.2007. 04.024

Fortin G, Champagnat J (1993) Spontaneous synaptic activities in rat nucleus tractus solitarius neurons in vitro: evidence for reexcitatory processing. Brain Res 630:125-135. doi:10.1016/ 0006-8993(93)90650-C

Herbert H, Moga MM, Saper CB (1990) Connections of the parabrachial nucleus with the nucleus of the solitary tract and the medullary reticular formation in the rat. J Comp Neurol 293:540-580. doi:10.1002/cne.902930404

Ji D, Wilson MA (2007) Coordinated memory replay in the visual cortex and hippocampus during sleep. Nat Neurosci 10:100-107. doi:10.1038/nn1825

Kalia M, Sullivan JM (1982) Brainstem projections of sensory and motor components of the vagus nerve in the rat. J Comp Neurol 211:248-265. doi:10.1002/cne.902110304

Kawai Y, Senba E (1996) Organization of excitatory and inhibitory local networks in the caudal nucleus of tractus solitarius of rats revealed in in vitro slice preparation. J Comp Neurol 373:309-321. doi:10.1002/(SICI)1096-9861(19960923)373:3< 309:AID-CNE1>3.0.CO;2-6

Kawai Y, Senba E (1999) Electrophysiological and morphological characterization of cytochemically defined neurons in the caudal nucleus of tractus solitarius of the rat. Neuroscience 89:13471355. doi:10.1016/S0306-4522(98)00393-5

Kawai Y, Senba E (2000) Postnatal differentiation of local networks in the nucleus of the tractus solitarius. Neuroscience 100:109-114. doi:10.1016/S0306-4522(00)00257-8

Lachamp P, Crest M, Kessler JP (2006) Vesicular glutamate transporters type 1 and 2 expression in axon terminals of the rat nucleus of the solitary tract. Neuroscience 137:73-81. doi: 10.1016/j.neuroscience.2005.08.048

Lau PM, Bi GQ (2005) Synaptic mechanisms of per sistent reverberatory activity in neuronal networks. Proc Natl Acad Sci USA 102:10333-10338. doi:10.1073/pnas.0500717102

Lübke J, Roth A, Feldmeyer D, Sakmann B (2003) Morphometric analysis of the columnar innervation domain of neurons connecting layer 4 and layer $2 / 3$ of juvenile rat barrel cortex. Cereb Cortex 13:1051-1063

McCann MJ, Rogers RC (1992) Impact of antral mechanoreceptor activation on the vago-vagal reflex in the rat: functional zonation of responses. J Physiol 453:401-411 
Okada T, Yoshioka M, Inoue K, Kawai Y (2006) Local axonal arborization patterns of distinct neuronal types in the caudal nucleus of the tractus solitarius. Brain Res 1083:134-144. doi: 10.1016/j.brainres.2006.02.026

Okada T, Tashiro Y, Kato F, Yanagawa Y, Obata K, Kawai Y (2008) Quantitative and immunohistochemical analysis of neuronal types in the mouse caudal nucleus tractus solitarius: focus on GABAergic neurons. J Chem Neuroanat 35:275-284. doi: 10.1016/j.jchemneu.2008.02.001

Ramon y Cajal S (1995) Histology of the nervous system of man and vertebrates, vol 1. Oxford University Press, New York, pp 589-615

Sanchez-Vives MV, McCormick DA (2000) Cellular and network mechanisms of rhythmic recurrent activity in neocortex. Nat Neurosci 3:1027-1034. doi:10.1038/79848

Shapiro RE, Miselis RR (1985) The central organization of the vagus nerve innervating the stomach of the rat. J Comp Neurol 238:473-488. doi:10.1002/cne.902380411

Shepherd GM, Stepanyants A, Bureau I, Chklovskii D, Svoboda K (2005) Geometric and functional organization of cortical circuits. Nat Neurosci 8:782-790. doi:10.1038/nn1447

Smith BN, Dou P, Barber WD, Dudek FE (1998) Vagally evoked synaptic currents in the immature rat nucleus tractus solitarii in an intact in vitro preparation. J Physiol 512:149-162. doi: 10.1111/j.1469-7793.1998.149bf.x

Stepanyants A, Chklovskii DB (2005) Neurogeometry and potential synaptic connectivity. Trends Neurosci 28:387-394. doi: 10.1016/j.tins.2005.05.006

Van der Kooy D, Koda LY, McGinty JF, Gerfen CR, Bloom FE (1984) The organization of projections from the cortex, amygdala, and hypothalamus to the nucleus of the solitary tract in rat. J Comp Neurol 224:1-24. doi:10.1002/cne.902240102
Watt AJ, Cuntz H, Mori M, Nusser Z, Sjöström PJ, Häusser M (2009) Traveling waves in developing cerebellar cortex mediated by asymmetrical Purkinje cell connectivity. Nat Neurosci 12:463-473. doi:10.1038/nn.2285

Weston M, Wang H, Stornetta R, Sevigny C, Guyenet P (2003) Fos expression by glutamatergic neurons of the solitary tract nucleus after phenylephrine-induced hypertension in rats. J Comp Neurol 460:525-541. doi:10.1002/cne.10663

Whitehead MC (1988) Neuronal architecture of the nucleus of the solitary tract in the hamster. J Comp Neurol 276:547-572. doi: 10.1002/cne. 902760409

Yoshioka M, Okada T, Inoue K, Kawai Y (2006) Pattern differentiation of excitatory and inhibitory synaptic inputs on distinct neuronal types in the rat caudal nucleus of the tractus solitarius. Neurosci Res 55:300-315. doi:10.1016/j.neures.2006.04.001

Yuste R, MacLean JN, Smith J, Lansner A (2005) The cortex as a central pattern generator. Nat Rev Neurosci 6:477-483. doi: $10.1038 / \mathrm{nrn} 1686$

Zhang X, Fogel R, Renehan WE (1995) Relationships between the morphology and function of gastric- and intestine-sensitive neurons in the nucleus of the solitary tract. J Comp Neurol 363:37-52. doi:10.1002/cne.903630105

Zhang X, Renehan WE, Fogel R (1998) Neurons in the vagal complex of the rat respond to mechanical and chemical stimulation of the GI tract. Am J Physiol 274:G331-G341

Zhou Z, Poon CS (2000) Field potential analysis of synaptic transmission in spiking neurons in a sparse and irregular neuronal structure in vitro. J Neurosci Methods 94:193-203. doi: 10.1016/S0165-0270(99)00144-2 